\title{
UNA CARTA CASI DESCONOCIDA DE CALDERÓN DE LA BARCA, Y EL TEMPLO DE PALAS DE FRANCISCO DE AVELLANEDA
}

Don W. Cruickshank

Department of Spanish University College Dublin

Belfield

Dublin 4 Irlanda

Don.Cruickshank@ucd.ie

[Anuario calderoniano (ISSN: 1888-8046), vol. extra, 1, 2013, pp. 83-96]

Francisco de Avellaneda de la Cueva y Guerra nació en Álava, en una familia noble, pero no sabemos exactamente cuándo: posiblemente alrededor de 1625, y parece que falleció en 1684. Escribió diez comedias, la mayor parte de ellas en colaboración, y más de treinta entremeses, mojigangas o bailes, algunos de los cuales salieron impresos en colecciones como Rasgos del ocio, Verdores del Parnaso, etc.; la Biblioteca Nacional conserva varios manuscritos suyos, algunos autógrafos. Entre éstos destaca una mojiganga titulada El titeretier (Ms. 15.164), representada en palacio el martes de Carnestolendas de 1663, por la compañía de Antonio de Escamilla, los miembros de la cual, entre ellos Manuela de Escamilla y Bernarda Manuela (ver abajo), aparecen nombrados en las acotaciones (es posible consultar este manuscrito en 
Internet, gracias a la Biblioteca Digital Hispánica). La primera acotación reza "Sale Escamilla de Alcalde ... cantando». Uno de los entremeses, El plenipapelier, impreso en 1664 en Rasgos del ocio, fue representado en la corte de Viena en abril de 1667 con Amado y aborrecido, de Calderón (algunas veces, los textos de las fiestas palaciegas vienesas salieron impresos en el mismo año, y en el caso de Fineza contra fineza, la obra principal iba acompañada de su loa y dos entremeses; pero si hubo una edición vienesa de Amado y aborrecido que incluía El plenipapelier, no ha llegado hasta nosotros). En Madrid, las cuentas de palacio indican que en febrero de 1676 Avellaneda recibió 1.650 reales por tres entremeses y un fin de fiesta, escritos todos para carnestolendas ${ }^{1}$. También consta que era canónigo de la catedral de Osma en 1669, y que actuó como censor de comedias en la corte. Aparte de los entremeses y mojigangas, parece que escribió por lo menos una obra palaciega más larga: El templo de Palas (1675); sin embargo, esta no es su única obra «real»: según los Avisos de Barrionuevo (30 de octubre de 1655), "la reina se muere por ver» la Vida y muerte de San Cayetano, escrita por seis ingenios (entre ellos, Avellaneda). La Inquisición la recogió, pero, gracias a la supuesta intervención de la reina, se licenció una versión modificada, que se estrenó en el corral del Príncipe al mes siguiente ${ }^{2}$; y parece que el rey vio La corte en el valle, escrita en colaboración con Matos y Villaviciosa, en Valladolid en $1660^{3}$.

En su calidad de censor de comedias, Avellaneda licenció las obras calderonianas La exaltación de la cruz (25 de octubre de 1662), El postrer duelo de España (29 de junio de 1668), Los tres mayores prodigios (el 1 de julio de 1669), El gran príncipe de Fez (15 de septiembre de 1669), El alcaide de sí mismo (24 de septiembre de 1669) y El mayor monstruo los celos (23 de abril de 1672). El gran príncipe de Fez era nueva, pero las otras habían sido representadas antes ${ }^{4}$. En ese mismo año también escribió una de las aprobaciones para la Cuarta parte de don Pedro, pero como este tomo fue editado por un amigo anónimo del autor, no sabemos quién le invitó a escribirla.

1 Shergold y Varey, 1982, pp. 72, 238.

2 Barrionuevo de Peralta, 1968-1969, vol. I, pp. 212, 214.

${ }^{3}$ Urzáiz Tortajada, 2002, vol. I, pp. 141-143.

${ }^{4}$ Wilson, 1961, pp. 172-174. 
La British Library tiene una enorme cantidad de comedias sueltas del Siglo de Oro español, muchas de ellas encuadernadas en tomos por coleccionistas del siglo XIX. A diferencia de algunas bibliotecas (por ejemplo, la London Library), la British Library no posee un catálogo dedicado únicamente a estas comedias, y los estudiosos tienen que buscarlas en el catálogo general; pero, como muchos volúmenes de la versión impresa de ese catálogo no son muy recientes, las fechas que sugieren para las ediciones no fechadas no son siempre fiables, y algunas desglosadas no han sido identificadas correctamente. Hace falta decir que el catálogo electrónico ha corregido la mayoría de estos errores, pero, como no es un catálogo descriptivo, no soluciona todos los problemas. Algunas veces el investigador no tiene más remedio que examinar los tomos en la biblioteca, o por medio de un microfilm.

Uno de los tomos a los que me refiero tiene la signatura T.1737. Contiene veintiséis obras: veinte sueltas y seis desglosadas. Siete de las sueltas son del siglo XVIII, y de poco interés, pero todas las desglosadas son del siglo XVII. Tres son de Calderón: El golfo de las sirenas, desglosada de su cuarta parte de 1672 («Madrid? 1730?», según el catálogo impreso); Lances de amor y fortuna, un fragmento de la Primera parte de 1636, de la que existen solo seis ejemplares completos («Valencia? 1735?», según el catálogo impreso); y Nunca lo peor es cierto, la segunda obra en Comedias escogidas I, de 1652: la primera edición. La cuarta es La gitana melancólica de Gaspar de Aguilar, desglosada de Doce comedias famosas de quatro poetas naturales de la insigne y coronada ciudad de Valencia, impresa en Madrid por Miguel Serrano de Vargas en 16145. La quinta es No se pierden las finezas, de Andrés de Baeza, impresa en Comedias escogidas XI en 1659, y la última es El marido asigurado de Carlos Boyl, una desglosable que formaba parte del Norte de la poesía española, impreso en Valencia por Felipe Mey en $1616^{6}$. Es de notar que el tomo también contiene una suelta de la primera versión de $\mathrm{La}$ vida es sueño, posiblemente de Sevilla, a mediados del siglo XVII, y el número 6 en la lista preparada por Germán Vega García-Luengos ${ }^{7}$ : este ejemplar es el único conocido. En total, el tomo contiene dieciséis

${ }^{5}$ La primera edición es de Valencia (1608), la segunda de Barcelona (1609); esta es la tercera.

${ }^{6}$ En Juliá Martínez, 1929, hay láminas de la portada (vol. I, p. LXIX) y de la desglosable (vol. I, p.CXXII).

${ }^{7}$ Calderón de la Barca, La segunda versión de "La vida es sueño», p. 78. 
obras genuinas de Calderón, y cuatro atribuidas. Parece que el responsable de la encuadernación se interesaba mucho por don Pedro, pero no sabemos quién fue. Se notará que los números 1 a 21 son obras "de Calderón», genuinas o atribuidas; los números 22 a 26 llevan los nombres de otros autores. Pongo aquí una lista completa, con colaciones:

1 Calderón, Las armas de la hermosura (Barcelona, Francisco Suriá, 1766). A-E .

2 Calderón, Amar después de la muerte (s.i., s.a. [¿Sevilla?, ¿alrededor de 1690?]). A-D ${ }^{4}$.

3 Calderón, Un castigo en tres venganzas (s.i., s.a. [¿Madrid?, ¿alrededor de 1685?]). A-D ${ }^{4}$.

4 Calderón, La gran Cenobia (Barcelona, Francisco Sapera, 1763). A$\mathrm{D}^{4}$.

5 Calderón, La crítica del amor [No hay burlas con el amor] (s.i., s.a. [¿Madrid?, ¿alrededor de 1685?]). A-D ${ }^{4}$.

6 Calderón, La desdicha de la voz (s.i., s.a. [¿Sevilla?, ¿alrededor de 1670 ?]). A-B ${ }^{8} \mathrm{C}^{4} \mathrm{D}^{2}$.

7 «Calderón", La dicha del retraído (s.i., s.a. [¿'Madrid?, ¿alrededor de 1685?]). A-D ${ }^{4}$.

8 Un ingenio, Los empeños de un plumaje [algunas veces atribuida a Calderón] (Valencia, Josef y Tomás de Orga, 1782). A-D ${ }^{4}$.

9 Calderón, El garrote más bien dado [El alcalde de Zalamea] (s.i., s.a. [¿Madrid?, ¿alrededor de 1680?]). A-D $\mathrm{E}^{2}$.

10 Calderón, El golfo de las sirenas (desglosada: pp. 493-516 de su Cuarta parte de 1672, p. 517 manuscrita).

11 Calderón, La hija del aire I (Barcelona, Carlos Sapera, 1763). A-D ${ }^{4}$ $\mathrm{E}^{2}$.

12 Calderón, La hija del aire II (Barcelona, Carlos Sapera, 1763). A-D ${ }^{4}$ $\mathrm{E}^{2}$.

13 «Calderón», El imposible más fácil (s.i., s.a. [Sevilla, Tomé de Dios Miranda, alrededor de 1675]). A-D ${ }^{4}$.

14 Calderón, Lances de amor y fortuna (desglosada: fols. $171 \mathrm{r}-194 \mathrm{v}$ de su Primera parte de 1636).

15 Calderón, Nunca lo peor es cierto [No siempre lo peor es cierto] (desglosada: fols. 17-38 de Escogidas I, Madrid, Domingo García Morrás, 1652, algunas hojas manuscritas); primera edición. 
16 Calderón, El secreto a voces (s.i., s.a. [¿Sevilla?, ¿alrededor de 1660?]). A-B ${ }^{8} C^{2}$.

17 «Calderón», Quien calla, otorga (s.i., s.a. [¿Sevilla?, ¿alrededor de 1670 ?]). A-B ${ }^{8}$.

18 Calderón, También hay duelo en las damas (s.i., s.a. [¿Sevilla?, ¿alrededor de 1670?]). A-D $\mathrm{D}^{4} \mathrm{E}^{2}$.

19 Calderón, También hay duelo en las damas (Barcelona, Carlos Sapera, 1764). A-E $\mathrm{E}^{4} \mathrm{~F}^{2}$.

20 Calderón, La vida es sueño (s.i., s.a. [¿'Sevilla?, ¿alrededor de 1650?]). Vega num. 6, único ejemplar conocido. A-D $\mathrm{D}^{2}$.

21 «Calderón», Celos no ofenden al sol (s.i., s.a. [¿Madrid?, ¿alrededor de 1670?]) (j con dos puntos en el texto). A-D $\mathrm{D}^{4} \mathrm{E}^{2}$.

22 Un ingenio [= José de Cañizares], El falso nuncio de Portugal (Valencia, viuda de José de Orga, 1764). A-D $\mathrm{D}^{4}$.

23 Gaspar Aguilar, La gitana melancólica (desglosada: fols. X5-X8, Y-Aa ${ }^{8}$ de Doce comedias famosas de cuatro poetas naturales de la insigne y coronada ciudad de Valencia, Madrid, Miguel Serrano de Vargas, 1614).

24 Andrés de Baeza, No se pierden las finezas (desglosada: fols. 177-197 de Escogidas XI, Madrid, Gregorio Rodríguez, 1659).

25 Francisco de Avellaneda, El templo de Palas (Nápoles, Geronimo Fasulo, 1675); con loa, entremés y mojiganga. Una zarzuela, estrenada el 26 de julio de 1675; única edición conocida. $\mathbb{S}^{4} \mathrm{~A}-\mathrm{B}^{8} \mathrm{C}^{6}$. 26 Carlos Boyl Vives de Canesma, El marido asigurado (desglosable, con colación propia, A-B ${ }^{8} \mathrm{C}^{12}$ (faltan tres hojas, A6, A7 y A8): de Norte de la poesía española, Valencia, Felipe Mey, 1616).

Me refiero a estas obras para indicar que se trata de una colección interesante, y no típica. Desde el punto de vista de la fecha de composición, parece probable que El templo de Palas sea la última obra del tomo, aparte de El falso nuncio de Portugal (Cañizares nació en 1676). Dos de ellas son fiestas reales: El golfo de las sirenas (calificada de "Égloga piscatoria» en la primera edición), y El templo de Palas de Avellaneda. La portada de esta reza: EL TEMPLO | DE PALAS, | COMEDIA FAMOSA, | CON SV LOA, ENTREMES, Y MOJIGANGA, | DE DON FRANCISCO DE AVELLANEDA. I Con que se celebrò en Madrid | EL AVGVSTO NOMBRE DE LA REYNA N ${ }^{a}$. S ${ }^{a} . \mid \mathrm{D}^{\mathrm{A}}$. MARIANA | DE AVSTRIA | EN EL SOLEMNE DIA DE SV GLORIOSA SANTA, I A los XXVI. de Iulio deste Año Santo 
M.DC. $L X X V^{\diamond}$. A pesar de lo de "comedia famosa", las portadas de las obras cortas llevan las palabras Para la Zarzuela del Templo de Palas. El texto de la loa y del entremés indica que estuvieron presentes la reina y su hijo, Carlos II. Las portadas no dicen dónde se representó, y el título no aparece entre las «representaciones palaciegas» de Shergold y Varey ${ }^{9}$; gracias a la acotación "Córrese la mutacion del teatro» (fol. 18v), sin embargo, podemos sospechar que se hizo en el Coliseo del Buen Retiro. Esta edición, la única, fue impresa en Nápoles ese mismo año por Geronimo Fasulo; como en otros casos, el impresor incluye la loa, La flor del sol, el entremés, El triunfo del vellocino (hay solo uno, porque la obra principal tiene solamente dos jornadas), y la mojiganga, El mundo novi. Gracias al manuscrito M.3800/25 de la Biblioteca Nacional, sabemos que la música fue compuesta en parte, tal vez en su totalidad, por el maestro Juan Hidalgo, compositor de la música de La púrpura de la rosa, Celos aun del aire matan, y otras obras calderonianas ${ }^{10}$. El magnífico manuscrito Guerra, preparado alrededor de 1680 por José Manuel de Guerra, «Escriptor de la R. Capill ${ }^{a}$ de su majestad», y conservado en la biblioteca de la Universidad de Santiago (Ms 265), también contiene algunos fragmentos. Es aún posible comprar por Internet una grabación de una de las canciones de la jornada primera, «Ay que sí, ay que no...!», interpretada en el estreno por la criada Mosqueta, y en la actualidad por la soprano canadiense Suzie Leblanc.

Si nos preguntamos por qué se imprimió la suelta en Nápoles, la dedicatoria lo explica: el mecenas de Avellaneda era don Antonio Pedro Álvarez Osorio Gómez Dávila y Toledo, marqués de Astorga y virrey de Nápoles; Geronimo Fasulo era uno de los impresores más conocidos de Nápoles, con una carrera de casi treinta años, durante la cual imprimió varios libros en español. Cuando salió la Tercera parte de Calderón en 1664, el amigo que la publicó, Sebastián Ventura de Vergara Salcedo, «dej[ó] a mi albedrío el elegirlas dueño» (palabras del mismo don Pedro); y en efecto el poeta eligió al mismo marqués, que por aquel entonces era virrey de Valencia. Sigue a la dedicatoria de

${ }^{8}$ El 26 de julio es el día de Santa Ana.

${ }^{9}$ Shergold y Varey, 1982.

${ }^{10}$ Vélez de Guevara, Los celos hacen estrellas, p. 208. 
Avellaneda otro documento: «Papel que escribió D. Pedro Calderón de la Barca [...] a D. Francisco de Avellaneda tocante a esta comedia»:

Entre otras molestias, señor, y amigo mío, con que mi poca salud me mortifica, fue la no menos sensible, la de verme obligado a no salir de casa aquellos días, que se dio al pueblo la fiesta, q[ue] V.m. escribió en celebridad del no[m]bre de la reina nuestra señora, que Dios guarde; y hallándome añadido a mis dolores el de haber perdido tan buena tarde, envié (no sin disculpada envidia de los que la lograron) a suplicar V.m. me hiciese favor de remitirme el borrador, para restaurar en parte la perdida del todo: que au[n]que es verdad, q[ue] el papel no puede dar de sí lo vivo de la represe[n]tació[n], lo adornado de los trajes, lo sonoro de la música, ni lo aparatoso del teatro; con todo eso, a los que tenemos alguna experiencia de cuanto desmerece fuera de su lugar este (nada dichoso) genero de estudios; nos es mas fácil, que a otros suplir con la imaginación la falta de la vista, y del oído; mayormente cuando lo escrito es tal, que por si se mantiene en tan merecida estimación, que no permite que nada se eche menos. Hela leído una, y muchas veces, con admiración de que en tan corta esfera quepan tantos primores, como una fábula no vulgar, reducida a representable metro, en tan elegante, y no afectado estilo, que bastará él solo a enriquecer la lengua castellana, aun cuando no tuviera en su colocación tan bien distribuida la vari[e]dad de los afectos, así en lo heroico de las lides, como en lo lícito de los amores; pues grave en las sentencias, sutil en los conceptos, y limpio en lo jocoso, no perdona nada a la precisión de los números siempre constantes, siempre dulces, y siempre armoniosos.

Vuélvosela a V.m. por cumplir la palabra de que lo volvería, pero con sentimiento de no quedarme con tan perfecto dechado, por si tal vez se me encargase a mí la celebridad de otro festivo día: los de V.m. aumente Nuestro Señor con las felicidades, que merece, y yo deseo.

De Casa, julio 30 de 1675.

De V.m.q.s.m.b.

D. Pedro Calderón de la Barca.

Como no se trata de una aprobación en el sentido estricto, podemos imaginarnos que cuando don Pedro la escribió, no sabía que se publicaría en la edición de la obra; es de esperar que Avellaneda le haya pedido permiso. Sin embargo, cuando afirma que ha leído la obra, cuando habla de los primores, de la elegancia del estilo, y del enriquecer de la lengua castellana, nos acordamos de la fraseología de al- 
gunas de sus aprobaciones, por ejemplo la escrita en 1650 para las Obras varias de Jerónimo de Cáncer:

... con todo eso le he leído con cuidado, y no hallo en él inconveniente que reparar; antes bien mucho que agradecer al estilo, en quien se hallan usados con agudeza y donaire los primores de la lengua castellana...

Menos típicos que las palabras de una aprobación son los datos personales: la referencia a la mala salud, que le obliga a quedarse en casa; la afirmación de que a los que tienen alguna experiencia de "este género de estudios es más fácil que a otros suplir con la imaginación la falta de la vista y del oído»; y, por último, la sugerencia de que si a don Pedro se le encargase la preparación de otra obra festiva, esta podría servirle de dechado. La frase "que se dio al pueblo» es otra indicación de que la obra se hizo en el Coliseo, porque los otros teatros reales eran teatros privados. Finalmente, la referencia al «borrador» es interesante: al terminar una obra, un dramaturgo solía dar el borrador al apuntador de la compañía que iba a representarla, a fin de que este sacase una copia en limpio para la representación; en teoría, devolvería el borrador al autor. Don Pedro supone que Avellaneda no tiene otra versión del texto (lo que es probable), y que necesitará el borrador para preparar otra copia en limpio para la prensa: por eso promete devolvérselo.

Cuando examinamos el texto de las tres piezas menores impresas en la suelta, vemos que los nombres en los repartos no son de los personajes, sino de los actores. Esto no era inusual: el fin de fiesta impreso en la suelta de lujo de Fieras afemina Amor (planeada para el cumpleaños de la reina en diciembre de 1671), da los nombres de ocho actores. En este caso, algunos (6) actuaron en todas las obras menores, algunos (5) en dos, y los otros (7) en una. La lista de todos es como sigue (en la segunda columna he puesto el nombre del autor de comedias, y la fecha):

[Antonio de] Escamilla Bernarda [Manuela] Blas [¿Polope?]

Carlos Vallejo

Damián [¿Arias?]

Fabiana [Laura]
* gracioso con Simón Aguado, 11/II/1675

* segunda dama, Manuel Vallejo, 11/II/1675

* segundas barbas, Simón Aguado, 11/II/1675

*segundo galán, Simón Aguado, 11/II/1675

$\star$

primera dama, Simón Aguado, 11/II/1675 
Heredia

primer galán, Simón Aguado, 11/II/1675

[¿Jerónimo de Heredia?]

[Juan de] Malaguilla

Juan Fernández

*arpista, Simón Aguado, 11/II/1675

Juan Francisco

en listas de febrero de 1673 y 1681

Jusepa de Prado

La Anaya

[María de Anaya]

La Santos [María Santos]

Manuela [de Escamilla]

María de Valdés

dama, Simón Aguado, 11/II/1675

*tercera dama, Simón Aguado, 11/II/1675

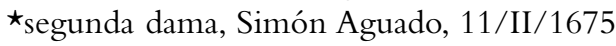

[mujer de Blas Polope]

Pablo [¿Polope?]

de por medio, Simón Aguado, 11/II/1675

[hijo de Blas Polope]

Salvador [¿de la Cueva?]

Simón Aguado

segundo gracioso, Simón Aguado, 11/II/1675

autor, 11/II/1675 [gracioso]

* en alguna de las otras listas de febrero de 1675, sin nombrar al autor de comedias

Los documentos preparados para la representación de los autos en 1675 facilitan casi todos estos detalles ${ }^{11}$. En ese año nadie quiso asumir la responsabilidad que conllevaba el encargarse de una de las dos compañías que los harían: Simón Aguado y Manuel Vallejo fueron detenidos, y tuvieron que pasar un tiempo en la Cárcel Real a causa de su renuencia. Finalmente, según un documento del 31 de mayo, se ofrecieron Escamilla y Vallejo. Es posible que las dos compañías se hayan combinado para hacer la fiesta real en julio. Tales combinaciones eran normales, sobre todo en una zarzuela: El templo de Palas tiene unos quinientos versos cantados (aproximadamente el 25\%), y no todos los actores de una compañía sabían cantar. Por esa misma razón es imposible, sin más datos, deducir cuáles desempeñaron los papeles de la obra principal. Sin embargo, otras fuentes nos dan más información: según la Genealogía, origen y noticias de los comediantes de España, María de Anaya, casada con José de Prado, «hizo quintas damas en Madrid en la compañia de Simon Aguado el año 1674», María (o «Mari») Santos, mujer de Pedro de Salazar, «fue célebre música», e «hizo

11 Shergold y Varey, 1961, pp. 284-286. En la nota 498 de la p. 296 se dan las cantidades pagadas a algunos de estos actores. 
quartas damas en Madrid en la compañia de Simon Aguado el año $1674 »^{12}$. Es probable que «Bernarda» sea Bernarda Manuela, por otro nombre «la Grifona», que "hizo... sobresaliente con el mismo autor [Vallejo] el año 1674» (p. 427). Blas Polope fue elegido mayordomo de la Cofradía de la Novena en 1676, «y estaba en la compañia de Antonio Escamilla» (p. 139). Fabiana Laura, después de tener su propia compañía, hizo primeras damas en la de Aguado en 1674 (p. 514). También leemos que "Juan de Malagilla [sic] el hijo estuvo en Valencia el año 1667 en la compañía de Antonio de Escamilla por tercer galan y arpista» (p. 197). Manuela de Escamilla era hijastra de Antonio de Escamilla, y «entre las muchas habilidades que ha tenido ha sido la mas celebrada la de la música» (p. 421). María de Valdés, segunda esposa de Blas Polope y madre de Pablo, «hizo segundas damas en Madrid en la compañía de Simón Aguado el año 1674» (p. 418). Jusepa de Prado era sin duda una actriz de la dinastía de los Prado, pero su nombre no consta en los documentos, ni en Genealogía. Finalmente, «Salvador» es probablemente Salvador de la Cueva, del cual se dice que «hizo graciosos y segundos» (p. 247). Manuel Vallejo, a pesar de ser un gracioso casi tan famoso como Escamilla, no actuó en las obras menores. En cuanto a éste, se sabe que recibió pagos por algunas obras cortas que parecen indicar que él las escribió; su carrera y sus "colaboraciones» con Calderón han sido documentadas por José Ruano ${ }^{13}$.

En el título de este artículo puse las palabras "casi desconocida» para describir la carta de Calderón; en efecto, no aparece entre los Documentos de Cristóbal Pérez Pastor, ni en el Ensayo de Cotarelo, ni entre las Cartas y documentos de Krzysztof Sliwa. Consta solo en el Manual de los Reichenberger ${ }^{14}$. La edición que la contiene es bastante rara: la Biblioteca Nacional posee tres ejemplares, y hay otros en Toledo, en Viena, y en la Biblioteca Palatina de Parma: siete en total. El único crítico que la ha utilizado - en busca de una definición del género «zarzuela»- es Louise Stein ${ }^{15}$. No podemos decir que se trata de un documento de suma importancia, pero tenemos muy pocas

12 Shergold y Varey (ed.), 1985, p. 474. Las citas que siguen son de la misma obra, se indicará las páginas correspondientes entre parentesis.

13 Calderón de la Barca, Cada uno para sí, pp. 19-24.

14 Pérez Pastor, 1905; Cotarelo y Mori, 1924; Sliwa, 2008; K. y R. Reichenberger, 1979-2002, vol. I, p. 714 (núm. 2857).

${ }^{15}$ Ver Stein, 1993 , pp. 259-261. Transcribe la mayor parte de la carta, con una traducción al inglés. 
cartas de Calderón: con ésta, son siete. Los datos personales que revela no son asombrosos. Es interesante notar que varios documentos relacionados con él se refieren a su "poca salud»: en otra carta, del 29 de abril de 1640, dice que terminará el auto sacramental que ha prometido, a pesar de su «poca salud»; en un documento escrito por Olivares para don Pedro el 15 de noviembre de 1642, el ministro se refiere a «achaques de calidad que le imposibilitan el continuar el real servicio» (al describir esta situación algunos años más tarde, el mismo Calderón utiliza las palabras "por hallarse muy enfermo»); y en una carta transcrita por Pérez Pastor, del 9 de octubre de 1648, el dramaturgo dice que "estoy en una cama con unas grandes tercianas» ${ }^{16}$. Finalmente, en la Verdadera quinta parte de 1682, refiriéndose a la lista incompleta de las obras no auténticas que puso don Pedro en su Cuarta parte diez años antes, Vera Tassis afirma que «su achacosa edad no permitió pudiese hacer entero juicio de sus comedias». No creo que debamos concluir que se trata de un caso de la enfermedad de Alzhéimer, sobre todo porque Gaspar Agustín de Lara lo contradice, insistiendo en que "hasta el último aliento de la vida, le conservó el cielo tan sano el juicio, que se desmintió humano"; sin embargo, las palabras de Vera Tassis podrían ser otra referencia a la «poca salud» física de Calderón» ${ }^{17}$. Dados los pocos documentos personales que tenemos, podríamos decir que esto es mucha enfermedad; pero es dificil saber si don Pedro era hipocondríaco, o si se trata de enfermedades verdaderamente graves. Las "grandes tercianas» podrían ser una fiebre palúdi$\mathrm{ca}$, es decir, malaria. La malaria era una enfermedad endémica en las zonas cálidas de Europa en el siglo XVII; efectivamente, una de las variedades se llama "malaria terciana» precisamente porque los síntomas se repiten cada tres días. Los jesuitas trajeron la quinina a Europa durante los años cuarenta del siglo XviI, pero la enfermedad puede volver a presentarse aun después de una "cura» aparentemente eficaz. Esto explicaría, quizá, las repetidas referencias a la falta de salud.

Finalmente, si don Pedro califica a Avellaneda de "amigo mío», no es fácil saber el grado de amistad que existía entre los dos. Lo que po-

16 Martínez Gil, García Ruipérez y Crosas, 2004, pp. 93-120; Picatoste, 1881, p. 20; Wilson, 1971, pp. 801-817; Pérez Pastor, 1905, pp. 161-162.

17 Lara, Obelisco fúnebre, pirámide funesto que construía a la inmortal memoria de D. Pedro Calderón de la Barca, fol. 8\1v. 
demos decir es que se conocían lo bastante bien para que Avellaneda le prestase a Calderón el borrador de la obra más importante que había escrito, y que cada uno estaba dispuesto a loar la obra del otro.

Es evidente, gracias a la edición suelta de El templo de Palas, que tenemos un detalle más de las fiestas palaciegas en la década de los setenta. Sin embargo, a pesar de las alabanzas de don Pedro, el drama resulta un poco decepcionante. La acción se sitúa en Atenas, con una guerra civil entre los hijos gemelos del rey Edipo, pero la trama es bastante floja; gracias a la intervención de los dioses, complacidos con la construcción del nuevo templo de Palas (es decir, el Partenón), los hermanos se reconcilian. Es posible argüir que el argumento refleja, hasta cierto punto, la situación que existía en España entre Juan José de Austria y la madre de su hermanastro Carlos II, pero no hay tiempo para el desarrollo de los personajes, o para investigar los sentimientos que los motivan. La palabra zarzuela se utiliza tres veces, en el encabezamiento de las obras menores, y si las palabras de don Pedro son una definición del género, una zarzuela es una fábula no vulgar, con heroicas lides, amores lícitos, sutil en los conceptos y limpia en lo jocoso - como todas las obras mitológicas calderonianas. Sin embargo, las intervenciones del gracioso Requesón (probablemente representado por Escamilla) y la criada Mosqueta (probablemente representada por Manuela de Escamilla, sobre todo porque tiene que cantar) son tan frecuentes que la obra podría calificarse de burlesca. Los chistes y juegos de palabras son, a veces, muy entretenidos. Por graciosas que sean, sin embargo, las piezas menores apenas reflejan los temas de la obra principal, como sucede, por ejemplo, en Fieras afemina Amor y El entremés del triunfo de Juan Rana, donde el triunfo del protagonista del entremés parodia la actitud triunfante de Hércules y prefigura el triunfo del amor sobre el supuesto héroe. Se podría decir que, en vez de crear un conjunto en el cual los temas de la obra principal influyen en las menores, Avellaneda ha hecho lo contrario: sus obras menores tienden a invadir la principal. En efecto, varios críticos han dicho que Avellaneda es un entremesista consumado; por eso, quizá, le resultó dificil resistirse a crear una obra que es más divertida que conmovedora ${ }^{18}$.

${ }^{18}$ Ver, por ejemplo, Urzáiz Tortajada, 2002, vol. I, p. 141. 


\section{BiBLIOGRAFÍA}

Avellaneda, F. de, El templo de Palas, Napoli, Geronimo Fasulo, 1675.

Barrionuevo de Peralta, J., Avisos, ed. A. Paz y Melia, Madrid, Atlas, 19681969, 2 vols.

Calderón de la Barca, P., Cada uno para sí, ed. J. M. Ruano de la Haza, Kassel, Reichenberger, 1982.

- La segunda versión de "La vida es sueño», ed. G. Vega García-Luengos, D. W. Cruickshank y J. M. Ruano de la Haza, Liverpool, Liverpool University Press, 2000.

Cotarelo y Mori, E., Ensayo sobre la vida y obras de D. Pedro Calderón de la Barca, Madrid, Tipografía de la "Revista de Archivos, Bibliotecas y Museos», 1924.

Juliá Martínez, E., Poetas dramáticos valencianos, Madrid, Tipografía de la «Revista de Archivos», 1929, 2 vols.,

LARA, G. A. de, Obelisco fúnebre, pirámide funesto que construía a la inmortal memoria de D. Pedro Calderón de la Barca, Madrid, Eugenio Rodríguez, 1684.

Martínez Gil, F., M. García Ruipérez y F. Crosas, "Calderón de la Barca y el Corpus toledano de 1640: recuperación de una carta autógrafa en el Archivo Municipal de Toledo", Criticón, 91, 2004, pp. 93-120.

Pérez Pastor, C., Documentos para la biografía de don Pedro Calderón de la Barca, Madrid, Fortanet, 1905.

Picatoste, F., «Biografia de Calderón», en Homenaje a Calderón, Madrid, Nicolás González, 1881, pp. 7-61.

Reichenberger, K. y R., Bibliographisches Handbuch der Calderón-Forschung / Manual bibliográfico calderoniano, Kassel, Thiele und Schwarz / Reichenberger, 1979-2002, 4 vols.

Shergold, N. D., y J. E. Varey, Los autos sacramentales en Madrid en la época de Calderón: 1637-1681. Estudio y documentos, Madrid, Ediciones de Historia, Geografia y Arte, 1961.

- (eds.), Genealogía, origen y noticias de los comediantes de España, London, Tamesis, 1985.

- Representaciones palaciegas: 1603-1699. Estudio y documentos, London, Tamesis, 1982.

Sliwa, K., Cartas, documentos y escrituras de Pedro Calderón de la Barca Henao de la Barrera Riaño (1600-1681) y de sus familiares, fénix de los ingenios y lucero mayor de la poesía española, Valencia, Universitat de València, 2008.

Stein, L. K., Songs of Mortals, Dialogues of the Gods: Music and Theatre in Seventeenth-Century Spain, Oxford, Clarendon Press, 1993.

Urzárz Tortajada, H., Catálogo de autores teatrales del siglo XVII. Investigaciones bibliográficas sobre autores españoles, Madrid, Fundación Universitaria Española, 2002, 2 vols. 
Vélez de Guevara, J., Los celos hacen estrellas, ed. J. E. Varey y N. D. Shergold, con una edición y estudio de la música por J. Sage, London, Tamesis, 1970.

WiLSON, E. M., «Calderón and the Stage-Censor in the Seventeenth Century: A Provisional Study», Symposium, 15, 1961, pp. 165-184.

— «Un memorial perdido de don Pedro Calderón», en Homenaje a William L. Fichter: Estudios sobre el teatro antiguo hispánico y otros ensayos, ed. A. D. Kossoff y J. Amor y Vázquez, Madrid, Castalia, 1971, pp. 801-817. 